\title{
Quantum fluctuations of mass for a mirror in vacuum
}

\author{
Marc-Thierry Jaekel ${ }^{a}$ and Serge Reynaud ${ }^{b}$ \\ (a) Laboratoire de Physique Théorique de l'ENS * 24 rue Lhomond F'75231 Paris Cedex 05 France \\ (b) Laboratoire de Spectroscopie Hertzienne de l'ENS ${ }^{\dagger}, 4$ place Jussieu F75252 Paris Cedex 05 France
}

(Physics Letters A 180 (1993) 9-14)

\begin{abstract}
A mirror in vacuum is coupled to fluctuating quantum fields. As a result, its energy-momentum and mass fluctuate. We compute the correlation spectra of force and mass fluctuations for a mirror at rest in vacuum (of a scalar field in a two-dimensional space-time). The obtained expressions agree with a mass correction equal to a vacuum energy stored by the mirror. We introduce a Lagrangian model which consistently describes a scalar field coupled to a scatterer, with inertial mass being a quantum variable.
\end{abstract}

\section{INTRODUCTION}

Quantum field fluctuations in vacuum lead to mechanical effects on objects by which they are scattered [1]. Two mirrors of a cavity scattering vacuum fields feel a radiation pressure (Casimir force), which results from a difference of field energy densities between the inside and outside of the cavity [2]. Casimir energy can be seen as a variation in the vacuum field energy stored in the cavity [3]. As known since Einstein [4], any energy stored in a box modifies the inertial mass of the box. A cavity moving in vacuum also feels an inertial force, in agreement with Einstein's principle of inertia of energy [5].

Casimir energy is the length dependent part of the vacuum energy stored by the cavity, i.e. the integral over frequency of the mean energy density of vacuum fields multiplied by the storage delay in the cavity [3. Scattering by a partially transmitting mirror also involves a non vanishing time delay which must lead to a stored energy. From equivalence between energy and mass, vacuum fluctuations are expected to induce a mass for a single mirror also. For a pointlike mirror scattering a scalar field in a two dimensional (2D) space-time, the relation between induced mass $(\langle\Delta m\rangle$ ) and time delay $(\tau)$ takes the form [3] (units are such that light's velocity is equal to $1 ; \hbar$ is Planck's constant):

$$
<\Delta m>=\int_{0}^{\infty} \frac{d \omega}{2 \pi} \hbar \omega \tau[\omega]
$$

The radiation pressure exerted on a mirror by a quantum field fluctuates, even in vacuum [6], []. Total energy and momentum remain conserved, whilst being continuously exchanged between mirror and field through fluctuations. As a result of energy-momentum conservation, an energy fluctuation absorbed by the mirror changes its rest energy [8]. Hence, the mirror's mass also exhibits fluctuations in vacuum. This can also be seen as a consequence of energy storage (1), since the energy density of vacuum fields is itself a fluctuating quantity.

In this letter, we derive correlation spectra associated with force and mass fluctuations for a mirror at rest in the vacuum of a (2D) scalar field. We use two equivalent descriptions of a partially transmitting mirror, in terms of a scattering matrix, and as a pointlike source for the field. The obtained spectra agree with expression (1) for the mean value of the mass induced by vacuum field fluctuations. When evaluated for a mirror at rest, expression (11) diverges. Actually, neglecting the mirror's recoil during scattering can only be consistent with an infinite mass. In order to remedy this defect, we introduce a local relativistic Lagrangian which takes recoil effects into account and entails conservation laws. The mirror's mass then appears as a quantum variable.

\section{FORCE AND MASS FLUCTUATIONS IN VACUUM}

For simplicity, we study the case of a pointlike mirror at rest (at position $q$ ), which scatters a scalar field $(\phi)$ in a (2D) space-time $\left(\left(x^{\mu}\right)_{\mu=0,1}=(t, x)\right)$. We first use a model previously introduced for describing partially transmitting mirrors [3, [7]. On each side of the mirror, the scalar field is the sum of two freely propagating components:

\footnotetext{
*Unité propre du CNRS associée à l'Ecole Normale Supérieure et à l'Université Paris-Sud

${ }^{\dagger}$ Unité de l'Ecole Normale Supérieure et de l'Université Pierre et Marie Curie associée du CNRS
} 


$$
\begin{aligned}
& \phi(t, x)=\varphi_{\text {in }}(t-x)+\psi_{\text {out }}(t+x) \quad \text { for } \quad x<q \\
& \phi(t, x)=\varphi_{\text {out }}(t-x)+\psi_{\text {in }}(t+x) \quad \text { for } \quad x>q
\end{aligned}
$$

The outcoming fields are linearly determined from the incoming fields by a scattering matrix (satisfying reality, causality and unitarity conditions):

$$
\left(\begin{array}{c}
\varphi_{\text {out }}[\omega] \\
\psi_{\text {out }}[\omega]
\end{array}\right)=S[\omega]\left(\begin{array}{c}
\varphi_{\text {in }}[\omega] \\
\psi_{\text {in }}[\omega]
\end{array}\right) \quad S[\omega]=\left(\begin{array}{cc}
s[\omega] & r[\omega] e^{-2 i \omega q} \\
r[\omega] e^{2 i \omega q} & s[\omega]
\end{array}\right)
$$

$s$ and $r$ are frequency dependent transmission and reflection amplitudes. We use the following notation for Fourier transforms:

$$
f(t)=\int_{-\infty}^{\infty} \frac{d \omega}{2 \pi} e^{-i \omega t} f[\omega]
$$

On both sides of the mirror, the field propagates freely and its stress tensor $\left(T^{\mu \nu}\right)$ is divergenceless:

$$
\begin{aligned}
& T^{00}=T^{11}=\frac{1}{2}\left(\partial_{t} \phi^{2}+\partial_{x} \phi^{2}\right) \\
& T^{01}=T^{10}=-\partial_{t} \phi \partial_{x} \phi
\end{aligned}
$$

The variation in time of the field energy-momentum $\left(P^{\mu}\right)$ is given by fluxes of the stress tensor through the mirror (difference between left and right sides):

$$
\begin{aligned}
& \dot{P}^{0}=-F^{0}=-\left\{\dot{\varphi}_{\text {in }}(t-q)^{2}-\dot{\psi}_{\text {out }}(t+q)^{2}\right\}+\left\{\dot{\varphi}_{\text {out }}(t-q)^{2}-\dot{\psi}_{\text {in }}(t+q)^{2}\right\} \\
& \dot{P}^{1}=-F^{1}=-\left\{\dot{\varphi}_{\text {in }}(t-q)^{2}+\dot{\psi}_{\text {out }}(t+q)^{2}\right\}+\left\{\dot{\varphi}_{\text {out }}(t-q)^{2}+\dot{\psi}_{\text {in }}(t+q)^{2}\right\}
\end{aligned}
$$

(at rest, $\dot{q}=0$; a dot stands for time derivative). In particular, the space component $F^{1}$ is the radiation pressure felt by the mirror and has already been studied [7]. For a single mirror and vacuum input fields, its mean value vanishes. However, because of quantum field fluctuations, the force fluctuates and its correlations do not vanish. The time component $F^{0}$ corresponds to an energy exchange, and will appear in the following as responsible for mass fluctuations. In a vacuum input state, the two field components are uncorrelated and have identical auto-correlations:

$$
<\varphi_{i n}[\omega] \varphi_{i n}\left[\omega^{\prime}\right]>=<\psi_{i n}[\omega] \psi_{\text {in }}\left[\omega^{\prime}\right]>=\frac{2 \pi}{\omega^{2}} \delta\left(\omega+\omega^{\prime}\right) \theta(\omega) \frac{1}{2} \hbar \omega
$$

( $\theta$ is Heaviside's step function). Force fluctuations on a mirror at rest are stationary:

$$
<F^{\mu}(t) F^{\nu}\left(t^{\prime}\right)>-<F^{\mu}><F^{\nu}>=C_{F^{\mu} F^{\nu}}\left(t-t^{\prime}\right)
$$

and are easily computed using Wick's rules and scattering matrix unitarity [7]:

$$
\begin{aligned}
C_{F^{\mu} F^{\nu}}[\omega] & =2 \hbar^{2} \theta(\omega) \int_{0}^{\omega} \frac{d \omega^{\prime}}{2 \pi} \omega^{\prime}\left(\omega-\omega^{\prime}\right) \alpha^{\mu \nu}\left[\omega^{\prime}, \omega-\omega^{\prime}\right] \\
\alpha^{00}\left[\omega, \omega^{\prime}\right] & =\operatorname{Re}\left\{1-s[\omega] s\left[\omega^{\prime}\right]-r[\omega] r\left[\omega^{\prime}\right]\right\} \\
\alpha^{01}\left[\omega, \omega^{\prime}\right] & =\alpha^{10}\left[\omega, \omega^{\prime}\right]=0 \\
\alpha^{11}\left[\omega, \omega^{\prime}\right] & =\operatorname{Re}\left\{1-s[\omega] s\left[\omega^{\prime}\right]+r[\omega] r\left[\omega^{\prime}\right]\right\}
\end{aligned}
$$

Correlation spectra in vacuum contain positive frequencies only (field excitations necessarily have positive energies with respect to vacuum). Force auto-correlations are positive and vanish at least as $\omega^{3}$ around zero frequency (vacuum energy-momentum is conserved on large time scales). Some properties still distinguish the two components of the force. In the limit of perfect reflection $(s=0, r=-1)$, fluctuations associated with the time component vanish, while radiation pressure fluctuations subsist and take a simple form [7]:

$$
\begin{aligned}
& C_{F^{0} F^{0}}[\omega]=0 \\
& C_{F^{1} F^{1}}[\omega]=\frac{\hbar^{2}}{3 \pi} \theta(\omega) \omega^{3}
\end{aligned}
$$


Actually, for a perfect mirror, $F^{0}$ vanishes as an operator while $F^{1}$ is twice the momentum density of the input field (see (5)). In the general case and at low frequencies, radiation pressure fluctuations exhibit the same behaviour while correlations of the time component actually behave as $\omega^{5}$ (as a consequence of scattering matrix unitarity, the first four coefficients in a quasistatic expansion around zero frequency vanish):

$$
\begin{aligned}
& C_{F^{0} F^{0}}[\omega] \sim\left\{\left(i s^{\prime}[0]\right)^{2}+\left(i r^{\prime}[0]\right)^{2}\right\} \frac{\hbar^{2}}{12 \pi} \theta(\omega) \omega^{5} \\
& C_{F^{1} F^{1}}[\omega] \sim r[0]^{2} \frac{\hbar^{2}}{3 \pi} \theta(\omega) \omega^{3}
\end{aligned}
$$

Both energy and momentum of the field exhibit fluctuations. At the quasistatic limit however, energy fluctuations are much less important than momentum fluctuations.

By conservation of total energy-momentum, $F^{\mu}$ is equal to the time derivative of the mirror's energy-momentum $p^{\mu}:$

$$
\dot{p}^{\mu}=F^{\mu}
$$

As a consequence, the mirror's energy and momentum also fluctuate and their correlation spectra are given by those of the force (6):

$$
C_{p^{\mu} p^{\nu}}[\omega]=\frac{1}{\omega^{2}} C_{F^{\mu} F^{\nu}}[\omega]
$$

For a mirror at rest, mass is equal to energy. Hence, mass fluctuates and its correlations in vacuum are readily obtained:

$$
C_{m m}[\omega]=\frac{1}{\omega^{2}} C_{F^{0} F^{0}}[\omega]
$$

At low frequency, mass fluctuations scale as $\omega^{3}$, and the mirror's mass can be considered as constant on a large time scale. The mass noise spectrum (which vanishes in the limit of perfect reflection) contains only positive frequencies in vacuum. Stationary correlations possessing this property correspond to non commutative variables, so that mass fluctuations have an irreducible quantum character.

\section{MIRROR AS A POINTLIKE SOURCE}

The scattering model for partially transmitting mirrors (equations (2) and (3)) amounts to consider that the mirror acts as a pointlike source (denoted by $J$ ) for the scalar field, which obeys equations of motion of the form:

$$
\left(\partial_{t}^{2}-\partial_{x}^{2}\right) \phi(t, x)-J(t) \delta(x-q)=0
$$

Different types of coupling are described by different functions for the source $J$ in terms of the field $\phi$. Equation (9) can be put in a form which expresses the energy-momentum balance between mirror and field ( $T^{\mu \nu}$ is the field energy-momentum tensor (4)):

$$
\partial_{\nu} T^{\mu \nu}=-F^{\mu} \delta(x-q) \quad F^{\mu}=-J \partial^{\mu} \phi(q)
$$

where $F^{\mu}$ is the force exerted by the field on the mirror. When $J$ depends linearly on the field $\phi$, force is a quadratic form of the field and coupling through radiation pressure is equivalent to the scattering model (5). Equation (9) corresponds to continuous boundary conditions for the field on the mirror, and the scattering coefficients take the following form:

$$
s[\omega]=1+r[\omega] \quad r[\omega]=-\frac{\Omega[\omega]}{\Omega[\omega]-i \omega}
$$

$\Omega$ is a causal positive function (analytic with $\operatorname{Re} \Omega>0$ for $\operatorname{Im} \omega>0$ ), even and real on the real axis $\left(\Omega[\omega]^{*}=\Omega[-\omega]=\right.$ $\Omega[\omega]$ for $\omega$ real), and describes the linear response of the source to the local field (field evaluated on the mirror):

$$
J[\omega]=-2 \Omega[\omega] \bar{\phi}[\omega] \quad \bar{\phi}(t)=\phi(t, q)
$$


From now on, we only consider the simple model which corresponds to instantaneous response of the source to the local field, $J=-2 \Omega \bar{\phi}$ with $\Omega$ constant. In this case, the force exerted by the field on the mirror takes a simple form:

$$
F^{\mu}=\partial^{\mu}\left(\Omega \phi^{2}\right)(q)
$$

showing (see (7) ) that there is a mass (energy at rest) correction for the mirror which identifies with a function of the local field:

$$
\Delta m=\Omega \bar{\phi}^{2}
$$

Positivity of the induced mass $(\Omega>0)$ can be seen as equivalent to causality of field scattering (11).

This simple model corresponds to a lorentzian reflectivity ( $\Omega$ playing the role of a high frequency reflection cut-off), with a frequency dependent reflection delay $\tau$ given by [3]:

$$
\operatorname{det} S[\omega]=e^{i \Delta[\omega]} \quad 2 \tau[\omega]=\partial_{\omega} \Delta[\omega]=\frac{2 \Omega}{\Omega^{2}+\omega^{2}}
$$

$(\Delta[\omega]$ is the sum of the two phase shifts at frequency $\omega)$. The correlation spectrum $((\mathbb{8})$ and $(6))$ can be rewritten in terms of the reflection delay only:

$$
C_{m m}[\omega]=2 \hbar^{2} \theta(\omega) \int_{0}^{\omega} \frac{d \omega^{\prime}}{2 \pi} \omega^{\prime}\left(\omega-\omega^{\prime}\right) \tau\left[\omega^{\prime}\right] \tau\left[\omega-\omega^{\prime}\right]
$$

Direct evaluation of the correlations of $\Delta m$ using (12) leads to the same expression (the field is continuous on the mirror and its value given by (2) and (3)):

$$
C_{m m}(t)=2 \Omega^{2} C_{\bar{\phi} \bar{\phi}}(t)^{2} \quad \Omega C_{\bar{\phi} \bar{\phi}}[\omega]=\theta(\omega) \hbar \omega \tau[\omega]
$$

It has the form of the correlation spectrum of the square of a gaussian variable whose mean value is given by (1) (which can also be obtained from (12)). The mean value and variance of the instantaneous mass are simply related:

$$
<\Delta m^{2}>-<\Delta m>^{2}=C_{m m}(0)=2<\Delta m>^{2}
$$

This relation shows that mass has important fluctuations on short time scales, although it remains practically constant in the low frequency domain:

$$
C_{m m}[\omega] \sim \frac{\hbar^{2}}{6 \pi} \theta(\omega) \frac{\omega^{3}}{\Omega^{2}} \quad \text { for } \quad \omega \ll \Omega
$$

As expected, mass fluctuations vanish in the limit of perfect reflection $(\tau=0)$. For non zero reflection delay:

$$
C_{m m}[\omega]=\frac{\hbar^{2}}{2 \pi} \theta(\omega) \frac{\Omega^{2}}{\omega\left(1+\frac{\omega^{2}}{4 \Omega^{2}}\right)}\left\{\left(1+\frac{\omega^{2}}{2 \Omega^{2}}\right) \log \left(1+\frac{\omega^{2}}{\Omega^{2}}\right)-\frac{\omega}{\Omega} \operatorname{tg}^{-1}\left(\frac{\omega}{\Omega}\right)\right\}
$$

Coupling a mirror to vacuum fields by radiation pressure modifies the dynamical nature of its rest energy or mass. Through its dependence on the field, the mirror's mass exhibits quantum fluctuations. Its mean value is also modified (11). For non zero reflection delay this expression is infinite, and diverges logarithmically (see (13)). Actually, the previous model gives a description which is consistent with momentum conservation only when the mirror has an infinite mass (frequency of the scattered field is conserved (3)). For a finite mass, recoil effects become important for incoming fields with sufficiently high frequency. It is also known that radiation pressure fluctuations make the mirror's position fluctuate [9]. In next section, we introduce a Lagrangian model which takes these effects into account.

\section{LAGRANGIAN MODEL OF A POINTLIKE SCATTERER}

We rewrite the quadratic coupling between field and scatterer in a relativistically invariant form by introducing a model Lagrangian. In a flat (2D) space-time, the classical action for a scalar field $(\phi)$ and a point mass $m$ (with time dependent position $q$ ), can be written:

$$
S=\int d^{2} x \frac{1}{2}(\partial \phi)^{2}-\int m\left(1-\dot{q}^{2}\right)^{\frac{1}{2}} d t
$$


which is equivalent to the following Lagrangian density:

$$
L=\frac{1}{2}\left[\partial_{t} \phi^{2}-\partial_{x} \phi^{2}\right]-m\left(1-\dot{q}^{2}\right)^{\frac{1}{2}} \delta(x-q)
$$

In accordance with the previous discussion, we consider that the scatterer's mass depends on the field:

$$
m=m_{b}+\Delta m
$$

where $m_{b}$ is a "bare" mass and $\Delta m$ is a (positive) scalar function of the local field $(\bar{\phi}) . \Delta m$ also represents the interaction of the field with sources located on the scatterer. Quadratic coupling corresponds to $\Delta m=\Omega \bar{\phi}^{2},(\Omega>0)$.

The resulting Euler-Lagrange equations are found to provide the field equations ((9) and (10)) with:

$$
J=-\left(1-\dot{q}^{2}\right)^{\frac{1}{2}} \frac{\delta m}{\delta \bar{\phi}}
$$

They also provide the equation of motion for the scatterer's position:

$$
\frac{d}{d t}\left(\frac{m \dot{q}}{\left(1-\dot{q}^{2}\right)^{\frac{1}{2}}}\right)=F^{1}
$$

The latter coincides with the usual equation of motion of a massive particle submitted to a force. However, it involves a mass which is no longer constant, but depends on the coupled field and on the particle's position. Total energymomentum is derived from Lagrangian (17) and is the sum of the field energy-momentum (given by (41)) and of the scatterer's energy-momentum (there is no additional interaction term) given by:

$$
p^{0}=\frac{m}{\left(1-\dot{q}^{2}\right)^{\frac{1}{2}}} \quad p^{1}=\frac{m \dot{q}}{\left(1-\dot{q}^{2}\right)^{\frac{1}{2}}} \quad\left(p^{0}\right)^{2}=\left(p^{1}\right)^{2}+m^{2}
$$

Energy-momentum conservation is obeyed (7). It follows that recoil is now included in the treatment of coupled mirror and field. The following inequality results from (20):

$$
\left|p^{1}\right| \leq\left|p^{0}\right| \quad \text { i.e. } \quad|\dot{q}| \leq 1
$$

The scatterer's velocity always remains smaller than light's velocity. The position of the scatterer follows a relativistic stochastic process which respects causality.

The scatterer's mass is no longer a parameter and must be treated as a quantum operator in the equation of motion (19). Mass exhibits quantum fluctuations due to its dependence on the scattered field. Furthermore, because of its dependence on the scatterer's position it does not commute with the scatterer's momentum or velocity. When neglecting the quantum fluctuations of position, the correlation spectrum of mass fluctuations is given by (14) or (16). This spectrum gives an approximation to mass fluctuations in the frequency domain where recoil effects can be neglected $(\hbar \omega \ll<m>)$. For low frequencies (smaller than the reflection cut-off $\Omega$ ), mass is practically constant (15), and equation of motion (19) is well approximated by the Newton law. Then, linear response formalism provides a consistent treatment of quantum fluctations of field and scatterer's position [9]. However, for higher frequencies $(\omega \sim \Omega)$, mass can no longer be considered as constant in (19), and its fluctuations must be taken into account. For frequencies comparable with the scatterer's mass $(\hbar \omega \sim<m>)$, recoil effects must be included. In particular, the scattering matrix differs from (3) for high frequencies. High frequencies contribute significantly to stored energy (1)), so that recoil effects must be accounted for when determining the induced mass.

\section{DISCUSSION}

Coupling a scatterer to quantum fields through radiation pressure results in modifications of its mass. A single mirror moving in vacuum experiences a motional force which contains an inertial term. The induced mass is the energy stored on vacuum fields due to scattering time delay, in conformity with the situation for a cavity [5]. In a cavity built from partially transmitting mirrors, storage times depend on the roundtrip time within the cavity and reflection delays on the mirrors. Both terms are known to contribute to Casimir energy [3]. Similarly, both terms contribute to the inertial force acting on the cavity as a whole. This means that vacuum fluctuations act as a Lorentz invariant source of inertia (in accordance with the principle of inertia of energy), not only for field energy stored within the cavity [5], but also for the energy associated with reflection delays. 
Motion in vacuum cannot remain causal and stable when the mass induced by field fluctuations becomes infinite 10]. As recoil and position fluctuations of the scatterer significantly influence field scattering at high frequencies, the energy stored on vacuum fields is expected to remain finite and smaller than the quasistatic mass (i.e $m_{b}>0$ in (18)). Then, motion caused by a force including radiative reaction of quantum fields can be shown to be described by a passive and therefore causal response function [11]. Mass generation through vacuum energy storage appears as a consistent way to entail causality and stability of motion in vacuum. Vacuum fluctuations are also known to contribute to the electron self energy. This must lead to fluctuations of the electromagnetic mass of the electron, that could be involved in a solution of the stability problem of electron motion [10].

Mass arising from vacuum fluctuations is a quantum variable. Mass fluctuations vanish on large time scales so that mass measured in the low frequency domain appears as a constant, as it should. On shorter time scales however (comparable with reflection delay), fluctuations of inertial mass become significant. From the principle of equivalence, gravitational mass is expected to exhibit the same fluctuations [12, thus backing up the necessity that gravitation also has quantum fluctuations 13 .

\section{Acknowledgements}

We acknowledge discussions with J.M. Courty, A. Heidmann, P.A. Maia Neto, J. Maillard and correspondence with G. Barton.

[1] Sciama D.W., in "The Philosophy of Vacuum" S.Saunders and H.R.Brown ed. (Clarendon 1991) p.137.

[2] Casimir H.B.G., Proc. K. Ned. Akad. Wet. 51 (1948) 793;

Plunien G., Müller B. and Greiner W., Phys. Rep. 134 (1986) 87.

[3] Jaekel M.T. and Reynaud S., J. Phys. I France 1 (1991) 1395.

[4] Einstein A., Annalen der Physik 19 (1906) 627.

[5] Jaekel M.T. and Reynaud S., J. Phys. I France 3 (1993) 1093.

[6] Barton G., J. Phys. A: Math.Gen. 24 (1991) 991; J. Phys. A: Math.Gen. 24 (1991) 5533; in "Cavity Quantum Electrodynamics" (Supplement: Advances in Atomic, Molecular and Optical Physics) P. Berman ed. (Academic Press, to appear).

[7] Jaekel M.T. and Reynaud S., Quantum Optics 4 (1992) 39.

[8] Einstein A., Annalen der Physik 18 (1905) 639.

[9] Jaekel M.T. and Reynaud S., J. Phys. I France 3 (1993) 1.

[10] Rohrlich F., Classical Charged Particles (Addison-Wesley, Reading, MA, 1965);

Dekker H., Phys. Lett. A 107 (1985) 255.

[11] Jaekel M.T. and Reynaud S., Phys. Lett. A 167 (1992) 227.

[12] Greenberger D.M., J. Math. Phys. 15 (1974) 395; Ann. Inst. Henri Poncaré 49 (1988) 307.

[13] De Witt B.S., J. Math. Phys. 3 (1962) 619;

Unruh W.G. in "Quantum Theory of Gravity" S.M. Christensen ed. (Bristol 1984) p.234. 